\title{
Barriers to adequate first aid for paediatric burns at the scene of the injury
}

\author{
Cody C. FreariD | Bronwyn Griffin' | Kerrianne Watt ${ }^{2}$ | Roy Kimble'
}

\begin{abstract}
Issue Addressed: The recommended first aid for burns, consisting of 20 minutes of cool running water (CRW) delivered within three hours of the injury, offers a simple yet effective means of improving health outcomes. The aim of this study was to determine patient and injury characteristics associated with inadequate CRW ther- apy among children with thermal burns, with the goal of identifying populations at greatest risk of undertreatment.

Methods: A cross-sectional study was performed on children treated at a large ter- tiary paediatric burns centre. First aid was evaluated as either "adequate" or "inade- quate", and then descriptive analyses were conducted to examine differences between the groups in age, ethnicity, location and socioeconomic status, among others.

Results: From 2013 to 2016, the families of 2522 patients were interviewed. Over- all, 31.3\% of children received adequate CRW at the scene of the injury. Provision of adequate CRW did not significantly differ with sex, ethnicity or nationality. Factors that were associated with inadequate first aid included very young age and early adolescence $(P<0.001)$, rural or remote location $(P=0.045)$, low socioeco- nomic status $(P=0.030)$, radiant heat and flame burns $(P<0.001)$, as well as burns occurring at recreational sites, on farm/trade/industrial properties and in the street $(P=0.001)$.

Conclusions: Although most burns occurred in close proximity to sources of CRW, first aid was poor across all demographics. The highest levels of undertreatment were found in children aged 0-2, adolescents aged 15-16, those living rurally or remotely, and the socioeconomically disadvantaged. So what? The study highlights the need for improved public education of first aid for burn injuries.

\section{KEY W ORD S}

adolescents, children, health education, injury

\section{1 | INTRODUCTION}

In Australia, burns represent a major cause of injury in paediatric populations, resulting in the hospitalisation of over 1400 children every year.' First aid delivered at the scene of the injury represents a simple, free and effective means of improving patient outcomes.

Current guidelines recommend at least 20 minutes of cool running water (CRW) applied to the burn site within three hours of the injury. ${ }^{2,3}$ Clinical benefits associated with this treatment include accelerated reepithelialisation, ${ }^{4,5}$ burns of more superficial depth, ${ }^{4}$ decreased rates of ICU admission ${ }^{6}$ and wound repair surgery, ${ }^{6-8}$ and shorter hospital stays, ${ }^{6,9}$ all of which may help to curb the significant
\end{abstract}


physical, psychological and financial burdens that burn injuries place on patients and their families. The demonstrated effects of CRW on healing might also reduce the risk of harmful long-term sequelae such as hypertrophic scarring.

Despite the easy accessibility of CRW in the majority of burn incidents, most Australian paediatric patients still fail to receive adequate first aid. In 2014, although 80\% of Australian and New Zealand children were administered some form of cooling following their injury, only $23 \%$ were given first aid consistent with the above guidelines. ${ }^{10}$ Factors that have previously been linked with suboptimal first aid include young age under 3.5 years, ${ }^{8,11}$ socioeconomic disadvantage ${ }^{8}$ and injuries caused by friction burns. ${ }^{4}$ Meanwhile, poor knowledge of first aid has been found to be more prevalent in parents with no first aid training or history of burns, ${ }^{12-14}$ non-English-speaking households ${ }^{12}$ and younger parents. ${ }^{15}$ The existing literature on barriers to first aid is limited, however, and restricted mainly to retrospective audits and questionnaire-based surveys.

The purpose of this large-scale, multiyear study was to investigate barriers to the delivery of CRW among children with thermal burns. Its scope was limited to first aid administered at the scene of the burn, as interventions provided in this setting are most likely to reflect the first aid practices of the general public (as opposed to paramedics or emergency department staff). Additionally, CRW appears to yield the greatest benefits if given immediately, ${ }^{16}$ lending particular significance to the actions taken by patients and their guardians in the period directly following the injury. The study aims to determine specific patient and injury characteristics associated with inadequate CRW therapy in order to identify the populations at greatest risk of undertreatment. Elucidation of specific risk factors for inadequate first aid at the point of injury will enable future public health campaigns to deliver a more targeted, effective message.

\section{2 | METHODS}

\subsection{Sample and setting}

A cross-sectional study was designed using the Queensland Paediatric Burns Registry (QPBR), a prospectively collected database containing information on patients treated at the Pegg Leditschke Children's Burns Centre (PLCBC) in the Lady Cilento Children's Hospital (LCCH). The PLCBC serves both inpatients and outpatients up to 16 years of age as the only tertiary paediatric burns clinic in Queensland.

Since July 2013 , every child undergoing care at the PLCBC has been documented in the QPBR. To be eligible for this study, patients had to present to the centre with a thermal injury between July 2013 and July 2016. Exclusion criteria included nonthermal burns for which cool running water has not been shown to provide a therapeutic benefit (ie, friction ${ }^{4}$ and chemical burns ${ }^{17}$ ), cases where the nature and/or duration of the first aid were unknown $(n=12)$ and patients with a permanent place of residence outside of Queensland $(\mathrm{n}=121)$.

\section{2 | Data collection}

All patients receiving care at the PLCBC were approached by an investigator at their first presentation to the centre. After obtaining consent from a legal guardian, investigators conducted a structured interview with each child and/or their family using a standardised digital proforma on an electronic device. The specific details collected from each patient are summarised in Table S1.

\section{3 | Definitions and categorisation}

Children were divided into two groups based on the adequacy of the first aid they received at the scene of the injury. For the purpose of this research, adequate first aid was defined as at least $20 \mathrm{~min}$ utes of cool running water delivered either continuously or cumulatively within three hours of the injury. Patients receiving any other form of aid not fitting this description, including CRW administered for less than 20 minutes duration and/or after three hours postburn, as well as alternatives such as aloe vera gel and ice, were placed in the inadequate group.

\section{4 | Data analysis}

Census-derived indexes developed by the Australian Bureau of Statistics were employed to indirectly assess the patients' socioeconomic status and remoteness through their residential postcode. The Accessibility/Remoteness Index of Australia' ${ }^{18}$ (ARIA) assigns a remoteness or accessibility value to every region in Australia, labelling them as either part of a major city, inner regional, outer regional, remote or very remote. In this study, the former two (major city and inner regional) and latter three (outer regional, remote and very remote) groups were combined to better identify any broader differences in the patterns of first aid between urban/suburban regions and more rural/remote locales. Measures of socioeconomic advantage and disadvantage were obtained from the Index of Relative Socioeconomic Advantage and Disadvantage ${ }^{19}$ (IRSAD). Deciles were created based on IRSAD scores and divided into three groups: disadvantaged (deciles 1-4), advantaged (deciles 5-8) and highly advantaged (deciles 9 and 10). These categories were selected both to compensate for the very small numbers in some deciles and, as with ARIA, to help determine larger trends.

Where variables were categorical, chi-square analyses were performed to examine differences between the groups across a range of demographic and injury-related factors (except where assumptions were violated, when Fisher's exact test was used). Chi-square tests for trend or independent samples $t$-tests were employed where the variables were ordinal or numerical, respectively.

\section{5 | Ethics}

The study was approved by the Children's Health Service DistrictHuman Research Ethics Committee (HREC/16/QRCH/61). Participation was fully voluntary, with consent for inclusion in the QPBR 
obtained at the time of data collection. Patients were deidentified during analysis to guarantee anonymity.

\section{3 | RESULTS}

Between July 2013 and July 2016, interviews were conducted with 2522 patients and/or their guardians, which constituted approximately $97 \%$ of all thermal burn cases seen by the PLCBC during this three-year period. Sample characteristics are shown in Table 1. The cohort had a median age of two years and male-to-female ratio of approximately $3: 2$. The most common mechanism of injury was scalds $(49.0 \%, n=1237)$, followed by contact burns $(44.6 \%$, $\mathrm{n}=1126)$, flame burns $(4.9 \%, \mathrm{n}=123)$ and radiant heat burns $(1.4 \%, n=36)$. Electrical burns, accounting for less than $0.1 \%(n=2)$ of cases, were insufficient in number to merit inclusion in the statistical analysis.

More than $68 \%(n=1715)$ of the patients had received adequate first aid by the time they presented to the burns clinic. At the scene of the injury, however, adequate CRW was given $31.3 \%(n=790)$ of the time. Nonetheless, over $92 \%$ of patients $(n=2335)$ were provided with some form of first aid (Figure 1). There was no significant difference in provision of adequate first aid between sexes $(P=0.808)$.

\section{1 | Age}

The adequate CRW group was significantly older, with a mean age of 4.26 years $(95 \% \mathrm{Cl}, 3.97-4.54)$, compared with only 3.74 years $(95 \% \mathrm{Cl}, 3.55-3.93)$ for the inadequate group $(P=0.003)$. Across all age groups, the patients least likely to receive adequate CRW belonged to the $0-2$ years $(27.4 \%, n=385)$ and $15-16$ years $(27.3 \%$, $\mathrm{n}=6 ; P=0.005)$ age groups. These same groups, however, recorded some of the highest proportions of overall first aid (Figure 2).

\section{2 | Ethnicity and nationality}

A total of 193 of the patients identified as Aboriginal and/or Torres Strait Islander. Approximately $26.4 \%(n=51)$ of these children received adequate CRW, which was not significantly different than the proportion of non-Indigenous patients who were adequately treated (29.6\%, $\mathrm{n}=566 ; P>0.05)$. Differences between Australianor New Zealand-born children $(29.5 \%, \mathrm{n}=587 ; P>0.05)$ and patients born elsewhere $(24.2 \%, n=23)$ were also found to lack statistical significance.

\section{3 | Geographical location}

Adequate CRW varied as a function of geographical remoteness. Of the 2177 who resided in a major city or inner regional area, $32.4 \%$ $(n=706)$ were provided adequate CRW, compared with $26.3 \%$ $(n=51)$ of the 194 children living in an outer regional or remote/ very remote region $(P=0.045)$.
TABLE 1 Injury and patient demographic characteristics by adequacy of first aid

\begin{tabular}{|c|c|c|c|}
\hline Variable & $\begin{array}{l}\text { Adequate } \\
\text { treated } \\
\mathrm{n}(\%)\end{array}$ & $\begin{array}{l}\text { In Inadequately } \\
\text { treated } \\
\text { n (\%) }\end{array}$ & $\begin{array}{l}\text { Significance } \\
\text { (P-value) }\end{array}$ \\
\hline \multicolumn{4}{|l|}{ Gender $^{\mathrm{a}}$} \\
\hline Female & $317(31.0)$ & $704(69.0)$ & 0.808 \\
\hline Male & $466(31.5)$ & $1013(68.5)$ & \\
\hline \multicolumn{4}{|l|}{ Ethnicity ${ }^{\mathrm{a}}$} \\
\hline Indigenous & $51(26.4)$ & $142(73.6)$ & 0.358 \\
\hline Non-Indigenous & $566(29.6)$ & $1347(70.4)$ & \\
\hline \multicolumn{4}{|l|}{ Country of birth ${ }^{\mathrm{a}}$} \\
\hline Australia or NewZealand & $587(29.5)$ & $1406(70.5)$ & 0.272 \\
\hline Other & $23(24.2)$ & $72(75.8)$ & \\
\hline \multicolumn{4}{|l|}{ Geographical location ${ }^{a}$} \\
\hline Major city/inner regional & $706(32.4)$ & $1471(67.6)$ & 0.045 \\
\hline Outer regional/remote & $51(26.3)$ & $143(73.7)$ & \\
\hline \multicolumn{4}{|l|}{ Mechanism of injury } \\
\hline Scald & $379(30.6)$ & $858(69.4)$ & $<0.001^{\mathrm{b}}$ \\
\hline Contact & $382(33.9)$ & $744(66.1)$ & \\
\hline Flame & $28(22.8)$ & $95(77.2)$ & \\
\hline Radiant heat & $1(2.8)$ & $35(97.2)$ & \\
\hline \multicolumn{4}{|l|}{ Place of injury ${ }^{\mathrm{a}}$} \\
\hline Home & $695(32.6)$ & $1438(67.4)$ & 0.001 \\
\hline Holiday & $46(31.5)$ & $11(68.5)$ & \\
\hline School & $11(28.2)$ & $28(71.8)$ & \\
\hline Industrial/trade/farm & $9(17.6)$ & $42(82.4)$ & \\
\hline Recreational/sporting & $15(16.0)$ & $79(84.0)$ & \\
\hline Street/other & $6(17.6)$ & $28(82.4)$ & \\
\hline \multicolumn{4}{|l|}{ Day of the week ${ }^{a}$} \\
\hline School day & $346(32.1)$ & $733(67.9)$ & 0.494 \\
\hline Weekend/holiday & $444(30.8)$ & $998(69.2)$ & \\
\hline \multicolumn{4}{|l|}{ Season $^{a}$} \\
\hline Summer & $230(33.3)$ & $460(66.7)$ & 0.54 \\
\hline Spring & $149(28.7)$ & $371(71.3)$ & \\
\hline Autumn & $232(31.8)$ & $497(68.2)$ & \\
\hline Winter & $179(30.9)$ & $401(69.1)$ & \\
\hline Mean (SD) & & Mean (SD) & \\
\hline $\begin{array}{l}4.26 \text { years } \\
(95 \% \mathrm{Cl}, 3.97-4\end{array}$ & & $\begin{array}{l}3.74 \text { years } \\
(95 \% \mathrm{Cl}, 3.55-3\end{array}$ & $\begin{array}{l}0.003 \\
3.93)\end{array}$ \\
\hline
\end{tabular}

aSome data were missing as follows: sex $(n=22)$; Indigenous status $(n=416)$; country of birth $(n=434)$; geographical location $(n=151)$; place of injury $(n=114)$; day of week $(n=1)$; and season $(n=3)$. ${ }^{b}$ Even with radiant heat burns excluded from the analysis, a chi-square test still produced a significant $P$-value of 0.021 .

\subsection{Socioeconomic status}

A significant association was found between inadequate first aid and socioeconomic status. Disadvantaged patients were less likely to receive 20 minutes of CRW $(27.9 \%, n=219)$ than children living in 


\section{Provision of first aid}

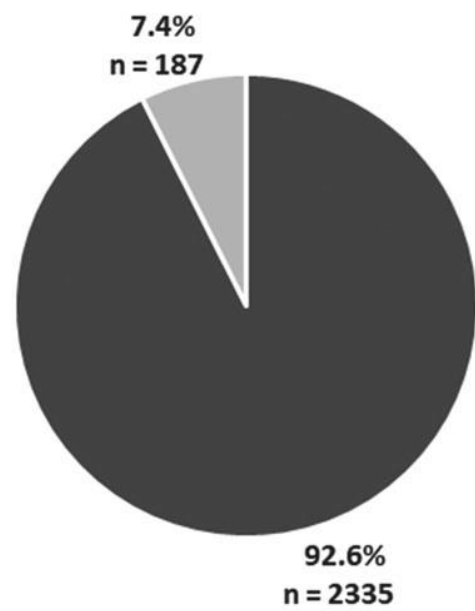

- Provided $=$ Not provided

\section{Adequacy of first aid}

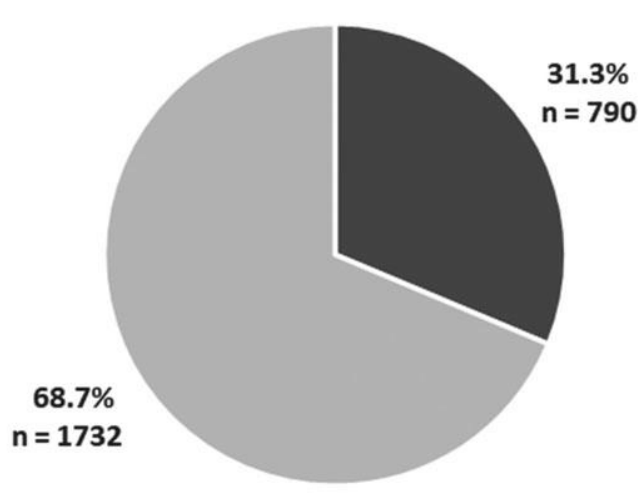

FI GU RE 1 Provision and adequacy of first aid at the scene of the injury. $\mathrm{N}=2522$
FI GU RE 2 Provision and adequacy of first aid by age. $\mathrm{N}=2519$

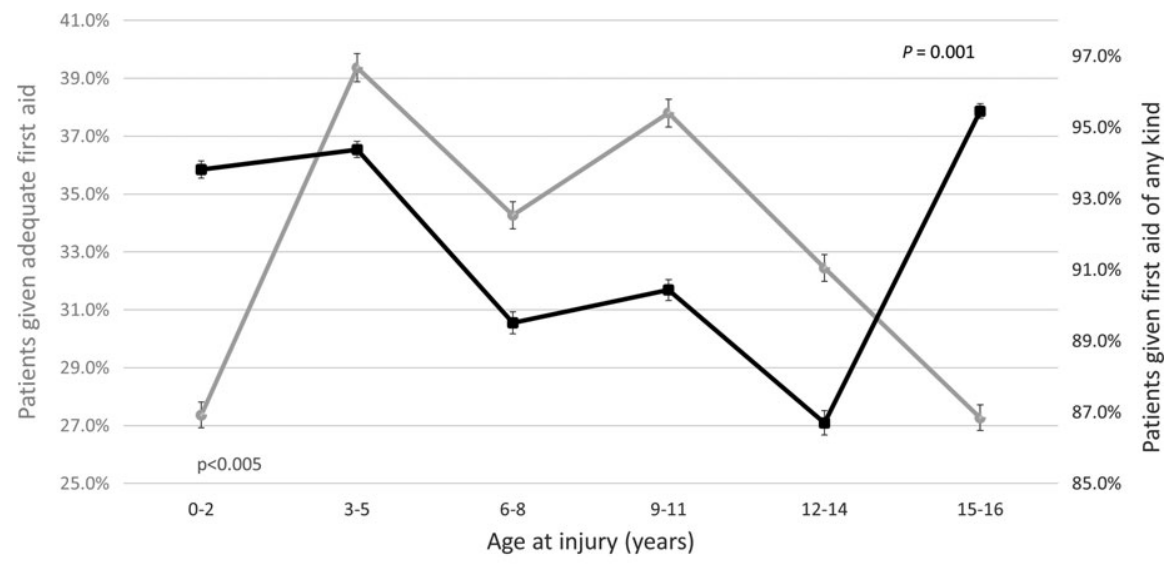

$\approx$ Adequate first aid ( $\geq 20$ min of CRW) $(n=790)$

- First aid of any kind (CRW of any length or alternatives) ( $n=2335$ ) advantaged $(33.4 \%, \mathrm{n}=375)$ or highly advantaged $(32.9 \%, \mathrm{n}=189)$ areas $(P=0.030)$ (Figure 3$)$.

\section{5 | Supervision}

Analysis of the types of supervision provided at the time of the injury yielded no discernible trends. Teachers were almost equally as likely to provide adequate first aid as parents. Siblings constituted the only group to deliver adequate CRW less than $25 \%$ of the time. The age of the sibling was not recorded in approximately $45 \%$ of cases, but the provided ages ranged from 8 to 28 years, with a mean age of 14.2 years $(95 \% \mathrm{Cl}, 12.6-15.8)$. Whether or not the burn injury was witnessed by a supervisor appeared to have no significant effect on subsequent first aid interventions $(P=0.227)$

\subsection{Mechanism of injury}

Scalds and contact burns were the best-treated of all burn types, receiving adequate CRW 30.6\% $(n=379)$ and 33.9\% $(n=382)$ of the time, respectively. They had a significantly greater likelihood of adequate first aid than flame-related $(22.8 \%, \mathrm{n}=28)$ and, especially, radiant heat $(2.8 \%, \mathrm{n}=1)$ burn injuries $(P<0.001)$.

\section{7 | Place of injury}

Burns that occurred in residential homes and schools, as well as those that took place on holiday, tended to be treated more adequately than in other settings, with over $25 \%$ of patients reporting adequate CRW. Patients were significantly less likely to be given adequate first aid if their burn injuries took place in the street $(17.6 \%, n=6)$, on agricultural or industrial properties $(17.6 \%, n=9)$, and at recreational/sporting sites $(16.0 \%, \mathrm{n}=15 ; P<0.05)$.

\section{8 | Timing of injury}

The likelihood of receiving adequate CRW did not vary significantly whether the burn occurred on a schoolday or weekend/holiday $(P>0.05)$, and there was negligible interseasonal variation $(P>0.05)$. 


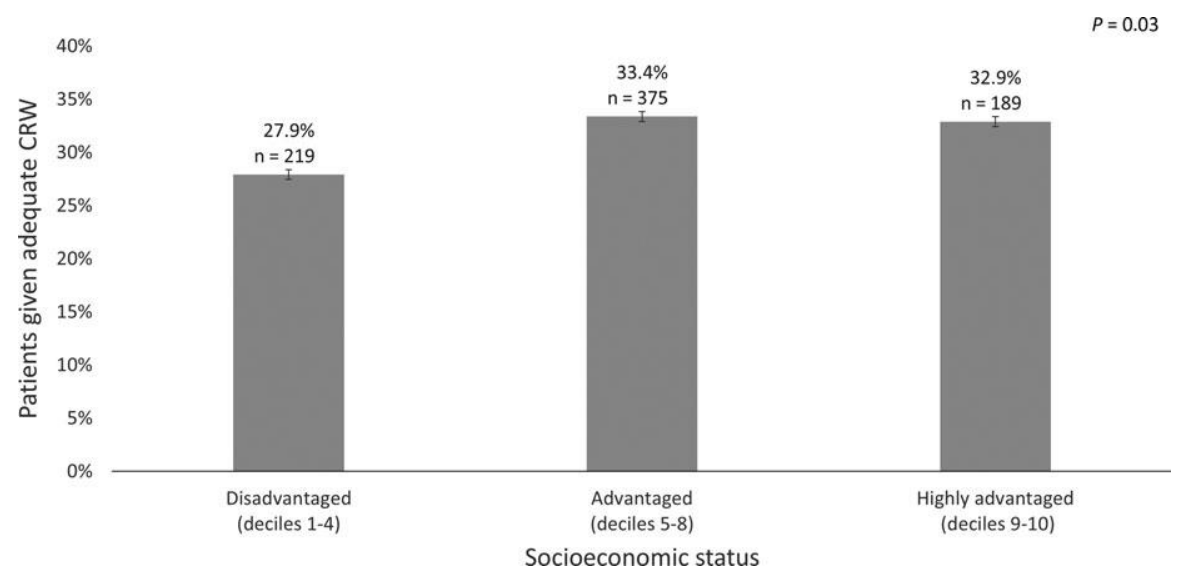

FIGURE 3 Provision of adequate first aid at the scene of the injury in children from different socioeconomic backgrounds. Deciles based on IRSAD scores. $\mathrm{N}=2484$

\subsection{Adequacy of CRW by year}

The duration of the study allowed for a broad comparison of adequate CRW across years. The highest annual proportion of patients given adequate first aid was recorded in 2013 (36.7\%, $n=235)$, the first year of the study. The following year marked a significant drop to $29.9 \%(n=209)$, followed by $28.8 \%(n=220)$ in 2015 and $30.1 \%$ $(n=126)$ in 2016 (Figure 4).

\section{4 | DISCUSSION}

In Australia, a child sustains a burn requiring transfer to a burns unit every six hours,' so the need for public awareness of appropriate first aid cannot be overstated. Relative to first aid practices for other common paediatric emergencies such as falls, road accidents and nonfatal drownings, optimal management of a thermal burn at the scene of the injury is particularly feasible for parents and children alike as it requires little training and an easily accessible resource in CRW. The overwhelming majority of paediatric burns occur in residential homes and schools, ${ }^{10}$ which would likely indicate easy access to a functioning tap. Therefore, it would appear that poor first aid practices documented by this study and others ${ }^{4,8,10}$ arise not necessarily from a lack of resources, but rather a lack of knowledge.
Public health campaigns addressing this knowledge gap could be a powerful health promotion approach. They could help ensure that more children receive treatment that can help mitigate the physical, financial and psychological costs associated with burn injuries and any subsequenthospitalisation and treatment. ${ }^{20}$

The present study identifies potential risk factors for inadequate first aid provision, highlighting the vulnerable populations that should be prioritised by any health promotion interventions. Consistent with past studies ${ }^{4,8,11}$ that identified being young as a risk factor for suboptimal first aid, patients aged 0-2 years were found to be among the least likely to receive adequate CRW. At the same time, the proportion of 0 - to 2 -year-olds given at least some form of first aid was among the highest in the cohort. This paradoxical trend suggests that, while guardians of those aged 0-2 nearly always attempt to administer first aid, in most cases they fail to provide the type or duration of care necessary to yield the greatest therapeutic benefits. One possible explanation is that guardians often begin to cool the burn, yet struggle to reach the full $20 \mathrm{~min}$ utes due to the difficulty of keeping a highly distressed, active infant under a stream of water for this period of time. Other potential contributing factors include a fear of causing hypothermia and an especially high deficit of first aid knowledge among younger parents. ${ }^{15}$

The same trend seen in the 0-2 age range was also observed in the cohort's oldest age bracket, 15-to 16-year-olds. Presumably,

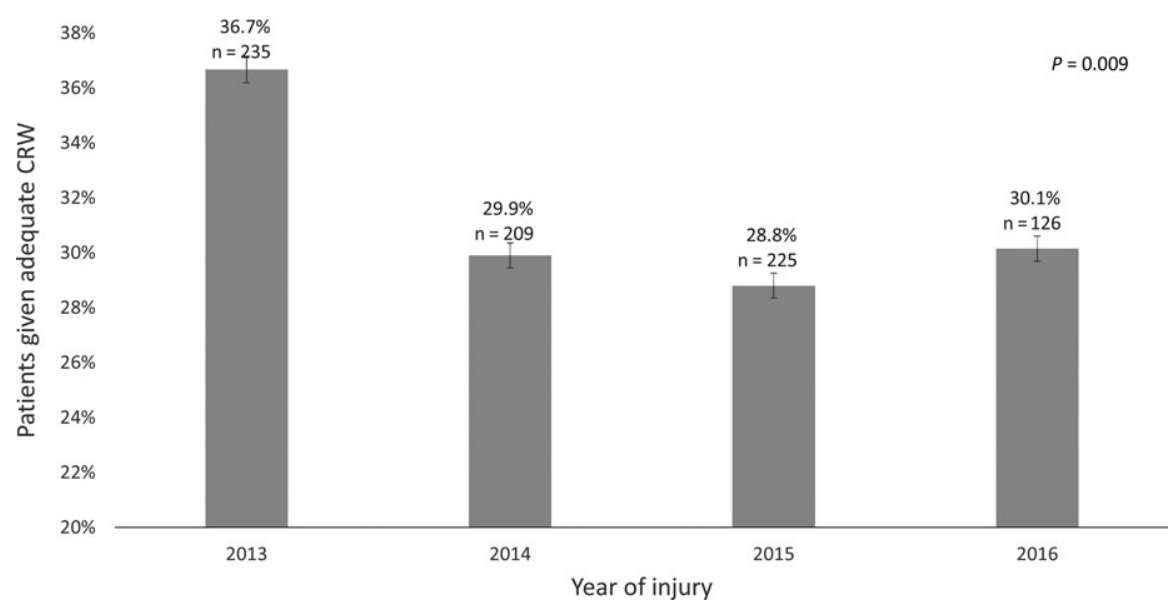

FIGURE 4 Proportion of patients who received adequate first aid at the scene of the injury by year. $\mathrm{N}=2522$ 
these patients have developed more independence and as a result are more likely to provide their own first aid, potentially increasing their probability of receiving at least some form of preliminary care. We hypothesise, however, that without the first aid experience of older guardians, the 15-to 16-year-olds are more liable to give themselves treatment of poorer quality.

Contrary to previous research ${ }^{8,12}$ that identified Indigenous and non-English-speaking backgrounds as risk factors for undertreatment and/or poor knowledge of appropriate first aid, likelihood of receiving adequate CRW did not vary significantly with ethnicity or nationality. Demographic features that were associated with suboptimal first aid included socioeconomic disadvantage and a residential address outside of a major city or inner regional area. Socioeconomic status was evaluated indirectly-through postcode, an admittedly imperfect proxy-but the connection between disadvantage and inadequate first aid has been described in the past. ${ }^{8,13}$ The reason behind disadvantaged and outer regional/remote patients receiving less adequate first aid is unclear, although more limited access to public health programs and first aid training may play a part.

Proportions of patients receiving adequate first aid remained fairly steady year-round, changing little with seasons and showing no significant difference between schooldays and weekends/holidays. This consistency suggests that children are no more susceptible to undertreatment at school than they are at home or on holiday. Indeed, parents and teachers appeared to provide roughly equal levels of first aid, and an analysis specifically of burn location found that injuries sustained in these settings were treated similarly. The places characterised by higher levels of inadequate first aid were, as expected, places where running water tends to be less immediately available-the street, sporting/recreational sites and farming/industrial properties. This kind of infrastructure barrier renders adequate first aid substantially more difficult, highlighting the significance of implementing burn prevention measures in locations less equipped with running water. Still, improved education on the importance of appropriate first aid might spur caregivers and/or children to seek out water even when a tap is not readily at hand.

Burn mechanism also had a significant impact on subsequent first aid treatment, with flame and radiant heat burns receiving substantially less adequate CRW than scalds and contact burns. Radiant heat burns in particular suffered from marked undertreatment. It is important to note that the effects of CRW have been less well studied in these burns than in other types of thermal injury; any therapeutic benefits that CRW first aid might confer have yet to be thoroughly investigated. Nevertheless, given that radiant burns are caused by heat-induced damage, with a histological appearance similar to that of other thermal burns, ${ }^{21}$ there is reason to believe that they might show a comparable response to CRW, although further investigation is required.

In the multiyear period covered by this study, annual measures of the proportion of paediatric patients provided adequate CRW revealed an alarming trend, dropping significantly from 2013 to 2015 before levelling off in 2016 . The initially higher numbers could be attributable, at least in part, to the public health campaign "Cool
Burns for 20," which was launched in 2011 to educate the Queensland public about appropriate first aid treatment. ${ }^{22}$ As time passed, reduced exposure to the campaign's message may have led to a general decrease in children's and guardians' awareness of CRW therapy. Regardless of its cause, however, the decline demonstrates that renewed efforts to improve public knowledge of first aid are warranted.

As these findings are based entirely on interviews conducted with families at their first presentation to an outpatient clinic, it is possible that some of the data may be biased (recall bias and/or selfreport bias), resulting in underestimation or overestimation of treatment length. Nevertheless, the structured interview format allowed for a more thorough investigation of the injury and subsequent management than a questionnaire or audit would have permitted. The study's other strengths include its large sample size, multiyear duration and diverse cohort comprised of children from various ethnic, socioeconomic and geographical backgrounds across the state of Queensland.

The study paves the way for further research, including multivariate analyses, to develop a more complete understanding of the factors contributing to the provision of inadequate first aid in the community. A follow-up study is currently underway to investigate the administration of CRW at later stages of preliminary treatment, following any interventions provided at the scene of the injury. The focus will be on potential interactions with ambulances, referral centres/hospitals and the LCCH emergency department, with the aim of assessing their effectiveness at recognising and rectifying prior lapses in burn management protocol.

\section{5 | CONCLUSION}

First aid consisting of 20 minutes of CRW is known to confer the greatest therapeutic benefits if given immediately following injury. ${ }^{16}$ This study highlights the need for improved initial management of burn injuries in children, as less than a third of paediatric patients received adequate first aid at the scene of the injury. Groups that were found to be particularly at risk for undertreatment included young children aged 0-2, adolescents aged 15-16, those living in rural or remote areas and the socioeconomically disadvantaged. Given the low proportions of patients receiving adequate CRW across all demographics, a public health campaign promoting awareness of appropriate first aid treatment would benefit the entire community, but programs targeting those particularly at-risk children and their guardians are a priority. Any future health campaigns should also emphasise the importance of CRW in the treatment of radiant heat and flame-related burns, as well as burns occurring in the street, at recreational/sporting sites and on industrial/trade/farm properties, which were all associated with suboptimal initial treatment. The general downward trend in annual measures of adequate CRW over the past three years accentuates the urgency of educating the public on first aid practices that can minimise the physical harm, psychological consequences and financial costs associated with paediatric burns. 


\section{CONFLICT OF INTERESTS}

The authors declare that there are no conflicts of interest in connection with this article.

\section{ORCID}

Cody C. Frear (iD http://orcid.org/0000-0002-6011-2472

\section{RE FE RE NC ES}

1. Pointer S, Tovell A. Hospitalised burn injuries, Australia, 2013-14. Canberra: Australian Institute of Health and Welfare Injury; 2016.

2. Cuttle L, Kimble RM. First aid treatment of burn injuries. Wound Pract Res. 2010;18(1):6.

3. Dyson S. Australian First Aid: An Authorised Manual of St John Ambulance Australia. 4th ed. Forrest, A.C.T.: St. John. Ambulance Australia; 2006

4. Cuttle L, Kravchuk O, Wallis B, Kimble RM. An audit of first-aid treatment of pediatric burns patients and their clinical outcome. J Burn Care Res. 2009;30:1028-34.

5. Frear C, Griffin B, Natsios C, Kimble R. The impact of cold running water first aid on paediatric burns and clinical outcomes. ANZJ Surg. 2017;87(S1):94.

6. Wood FM, Phillips M, Jovic T, Cassidy JT, Cameron P, Edgar DW. Water first aid is beneficial in humans post-burn: evidence from a binational cohort study. PLoS ONE. 2016;11(1):e0147259.

7. Nguyen NL, Gun RT, Sparnon AL, Ryan P. The importance of immediate cooling -a case series of childhood burns in Vietnam. Burns. 2002;28:173-6.

8. Skinner A, Peat B. Burns treatment for children and adults: a study of initial burns first aid and hospital care. N Z Med J 2002;115:U199.

9. Riedlinger DI, Jennings PA, Edgar DW, et al. Scald burns in children aged 14 and younger in Australia and New Zealand-an analysis based on the Burn Registry of Australia and New Zealand (BRANZ). Burns. 2015;41:462-8.

10. Dyson K, Gabbe B, Thomas T. Burns registry of Australia and New Zealand annual report. Melbourne: Australian and New Zealand Burns Association; 2016.

11. Burlinson CE, Wood FM, Rea SM. Patterns of burn injury in the preambulatory infant. Burns. 2009;35(1):118-22.

12. Harvey LA, Barr ML, Poulos RG, Finch CF, Sherker S, Harvey JG. A population-based survey of knowledge of first aid for burns in New South Wales. Med J Aust. 201 1;195:465-8.

13. Davies M, Maguire S, Okolie C, Watkins W, Kemp AM. How much do parents know about first aid for burns? Burns. 2013;39:1083-90.

14. Alomar M, Al Rouqi F, Eldali A. Knowledge, attitude, and belief regarding burn first aid among caregivers attending pediatric emergency medicine departments. Burns. 2016;42:938-43.
15. Gamelli L, Mykychack I, Kushnir A, Driscoll DN, Fuzaylov G. Targeting burn prevention in Ukraine: evaluation of base knowledge in burn prevention and first aid treatment. J Burn Care Res. 201 5;36 (1):225-31.

16. Cuttle L, Kempf M, Liu PY, Kravchuk O, Kimble RM. The optima duration and delay of first aid treatment for deep partial thickness burn injuries. Burns. 2010;36:673-9.

17. Walsh K, Stiles K, Dheansa B. Letter in Response to: European Resuscitation Council's guidelines for resuscitation 2015. Resuscitation. 2016;99:e13.

18. Accessibility/Remoteness Index of Australia (ARIA+) [Internet]. Adelaide: Hugo Centre for Migration and Population Research, The University of Adelaide; 2013 [cited 2017 Feb 18]. Available from: http://www.spatialonline.com.au/ARIA_2011/ default.aspx

19. Census of Population and Housing: The Index of Relative Socio-Economic Advantage and Disadvantage [Internet]. Canberra: Australian Bureau of Statistics; 2013 [cited 2017 Feb 20]. Report no. 2033.0.55.001. Available from: http://www.abs.gov.au/AUSSTATS/ subscriber.nsf/log?openagent $\& 2033.0 .55 .001 \% 20 \mathrm{POA} \% 20$ Indexes. xls\&2033.0.55.001\&Data\%20Cubes\&209B3364525C82CCCA257B3 B001A4D56\&0\&2011\&12.11.2014\&Latest

20. Skinner AM, Brown TL, Peat BG, Muller MJ. Reduced hospitalisation of burns patients following a multi-media campaign that increased adequacy of first aid treatment. Burns. 2004;30(1): 82-5.

21. Gurfinkel R, Singer AJ, Cagnano E, Rosenberg L. Development of a novel animal burn model using radiant heat in rats and swine. Acad Emerg Med. 201 0;1 7:514-20.

22. O'Donnell L. Education Campaign for First Aid Treatment of Burns [Internet]. Centre for Children's Burns and Trauma Research. 2011 [cited 24 March 2017]. Available from: https:// www.coolburns.com.au/news/42-education-campaign-for-first-aidtreatment-of-burns

\section{SUPPOR TING INFORMATION}

Additional supporting information may be found online in the Supporting Information section at the end of the article.

How to cite this article: Frear CC, Griffin B, Watt K, Kimble R. Barriers to adequate first aid for paediatric burns at the scene of the injury. Health Promot J Austral. 201 8;29:160166. https://doi.org/10.1002/hpja.184 